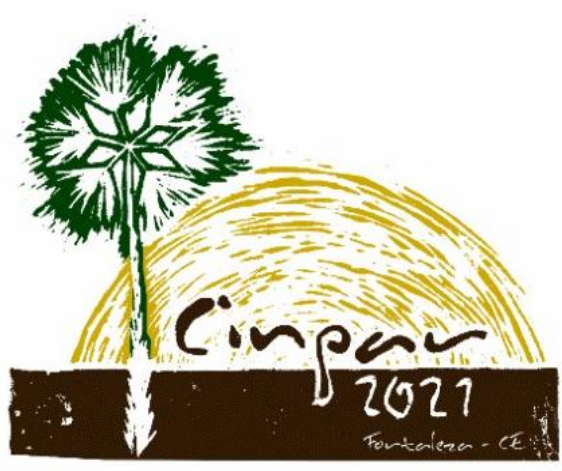

XVII Congresso Internacional sobre Patologia e Reabilitação das Construções

XVII Congreso Internacional sobre Patología y Rehabilitación de las Construcciones

XVII International Conference on Pathology and Constructions Rehabilitation

FORTALEZA (Brasil), 3 a 5 de junho de 2021

https://doi.org/10.4322/CINPAR.2021.115

\title{
Identificación y análisis de las manifestaciones patológicas en el Bloque E de la Universidad Privada del Valle, Bolivia
}

\section{Identification and analysis of pathological manifestations in Block $E$ of the Universidad Privada del Valle, Bolivia}

\author{
Giovana Silvia CACHACA-TAPIA ${ }^{1}$, Valeria Coral RODRIGUEZ-GARCIA², Nahúm Gamalier CAYO-CHILENO ${ }^{3}$, \\ Joaquin Humberto Aquino ROCHA ${ }^{4}$, Oscar AVILES-JIMENEZ ${ }^{5}$, Sergio Manuel RODRIGUEZ-BELMONTE ${ }^{6}$

\footnotetext{
${ }^{1}$ Universidad Privada del Valle, Tiquipaya, Bolivia, g.cachacatapia@gmail.com

${ }^{2}$ Universidad Privada del Valle, Tiquipaya, Bolivia, valeria.coral.rodriguez@gmail.com

${ }^{3}$ Universidad Privada del Valle, Tiquipaya, Bolivia, nahum.cayo.chileno@gmail.com

${ }^{4}$ Universidad Privada del Valle, Tiquipaya, Bolivia, joaquinaquinorocha@gmail.com

${ }^{5}$ Universidad Privada del Valle, Tiquipaya, Bolivia, oavilesj@univalle.edu

${ }^{6}$ Universidad Privada del Valle, Tiquipaya, Bolivia, srodriguezb@univalle.edu
}

\begin{abstract}
Resumen: A pesar de los avances tecnológicos en la construcción civil, todavía se reporta la presencia de manifestaciones patológicas en diferentes edificaciones, incluyendo las instituciones de educación superior. La falta de mantenimiento y el descuido por parte de la comunidad universitaria provocan el deterioro progresivo de las estructuras. El presente artículo tiene como objetivo identificar las principales manifestaciones patológicas encontradas en la tercera planta del Bloque E de la Universidad Privada del Valle, Bolivia. La metodología consistió en tres etapas: en la primera se delimitó el área a ser estudiada; la segunda consistió en una inspección visual y un levantamiento de las manifestaciones patológicas por medio de un registro fotográfico y un checklist, y en la tercera se realizó una descripción de los principales problemas encontrados para determinar el origen y las causas. Los resultados muestran que las principales manifestaciones patológicas son el deterioro de cielo falso, desprendimiento de revestimiento de yeso y pérdida de adherencia entre las piezas cerámicas y los pisos. Muchos de los problemas encontrados se atribuyen a la edad de la estructura y la falta de mantenimiento. Si bien no existe un riesgo estructural, es necesaria una evaluación integral para generar un programa de intervención a corto plazo.
\end{abstract}

Palabras clave: Inspección visual, causas, desprendimiento.

Abstract: Despite technological advances in civil construction, the presence of pathological manifestations is still reported in different buildings, including higher education institutions. The lack of maintenance and the neglect by the university community cause the progressive deterioration of the structures. This article aims to identify the main pathological manifestations found on the third floor of Block E of the Universidad Privada del Valle, Bolivia. The methodology consisted of three stages: in the first, the area to be studied was delimited; the second consisted of a visual inspection and a survey of the pathological manifestations by means of a photographic record and a checklist, and in the third a description of the main problems encountered was made to determine the origin and causes. The results show that the main pathological manifestations are the deterioration of the false ceiling, detachment of the plaster coating and loss of adhesion between the ceramic pieces and the floors. Many of the problems found are attributed to the age of the structure and the lack of maintenance. Although there is no structural risk, a comprehensive evaluation is necessary to generate a short-term intervention program.

Keywords: Visual inspection, causes, detachment. 


\section{Introducción}

La patología de las construcciones se define como el estudio de los mecanismos, las causas y el origen de los múltiples defectos de las edificaciones (Helene, 2015). Las manifestaciones patológicas evolucionan y se agravan en el tiempo, además de asociarse al surgimiento de otras. En este sentido, es importante la identificación temprana de los problemas a fin de realizar correcciones adecuadas y evitar, de esta forma, mayores costos en la intervención (Oliveira, 2013).

Entre las causas más comunes para el surgimiento de manifestaciones patológicas se mencionan: el medio en el que se encuentra situada la construcción, esfuerzos a los que se somete la estructura, mala elección de materiales, poca calidad del proyecto y el tipo de ejecución; sin embargo, también se generan durante la utilización. En esta última etapa, se deben realizar inspecciones y trabajos de mantenimiento con la finalidad de garantizar la vida útil de la construcción (Husni, 2007).

La vida útil de una edificación se define como el tiempo, una vez en utilización, en el cual las propiedades de la construcción están por encima de los niveles mínimos aceptables, considerando un mantenimiento periódico (American Society for Testing and Materials [ASTM], 1981). La vida útil dependerá, en cierta medida, en evitar someter a la construcción a condiciones no previstas a lo largo del proceso constructivo (planificación, proyecto, materiales, construcción, uso y mantenimiento) (Fernández, 1994).

Las obras civiles tienden a presentar una serie de manifestaciones patológicas (Antunes, 2010). En un estudio de Garza (2000) se reporta que, de los casos con manifestaciones patológicas, el $80 \%$ se debe a que muchos de los ingenieros encargados solo manejaban conceptos de su especialidad, lo que impedía que existiera complementariedad y se consideren aspectos que puedan generar problemas posteriores. Sin embargo, la presencia de las manifestaciones patológicas es inevitable dentro de una obra civil, es necesario tomar en cuenta previsiones para garantizar un adecuado rendimiento de la edificación durante su vida útil (Tutikian y Pacheco, 2013). Silva et al. (2017) indican que es urgente crear y divulgar herramientas adecuadas para desarrollar estrategias de mantenimiento, a través de inspección, recolección de datos, clasificación de anomalías y factores que contribuyan al diagnóstico y reparación.

Actualmente, el estudio de manifestaciones patológicas adquiere relevancia progresiva, puesto que muchas obras civiles presentan deterioro y daño, lo que puede llevar al colapso de las edificaciones (QuinteroQuintero et al., 2013). Esto adquiere mayor importancia en instituciones educativas, las cuales, por su función, tienen un alto contenido de valor humano, social y cultural, requiriendo mayor grado de seguridad (Ministerio de Obras Públicas, Servicios y Vivienda [MOPSV], 2006). Por tanto, el objetivo principal de la presente investigación es identificar las principales manifestaciones patológicas en el Bloque $\mathrm{E}$ de la Universidad Privada del Valle, Bolivia por medio de una inspección visual y registro fotográfico.

\section{Metodología}

\subsection{Caracterización del área estudiada}

El edificio seleccionado fue el Bloque E de la Universidad Privada del Valle, de finalidad académica y administrativa. Se ubica en el Campus Tiquipaya de la ciudad de Cochabamba, Bolivia. Las coordenadas de la edificación son: 794815.84 E y 8081706.38 S, zona 19K (Figura 1).

El Bloque E tiene 31 años de funcionamiento y está conformado por tres plantas, superficie total de 4699.10 $\mathrm{m}^{2}$. Sin embargo, debido a que las dos primeras plantas son utilizadas con fines administrativos, sólo se inspeccionó la tercera planta (Figura 2).

La tercera planta tiene un área de $1351.46 \mathrm{~m}^{2}$ y cuenta con 16 ambientes, distribuidos en aulas, oficinas, baños, auditorios y pasillos.

\subsection{Proceso de inspección}

El estudio de las manifestaciones patológicas tiende abordar una serie de temáticas de modo que llega a ser amplio y diverso (Harris, 2001). El presente estudio se delimitó solo para aquellos ítems encontrados en los 
ambientes internos del Bloque E y, específicamente, en la tercera planta. La investigación se desarrolló por medio de 3 etapas. La primera consistió en identificar el tipo de estructura. Para ello, fue necesario realizar un análisis significativo del edificio a ser estudiado y obtener, por medio de la visualización, un reconocimiento de la importancia y uso (Pallarés, 2007).

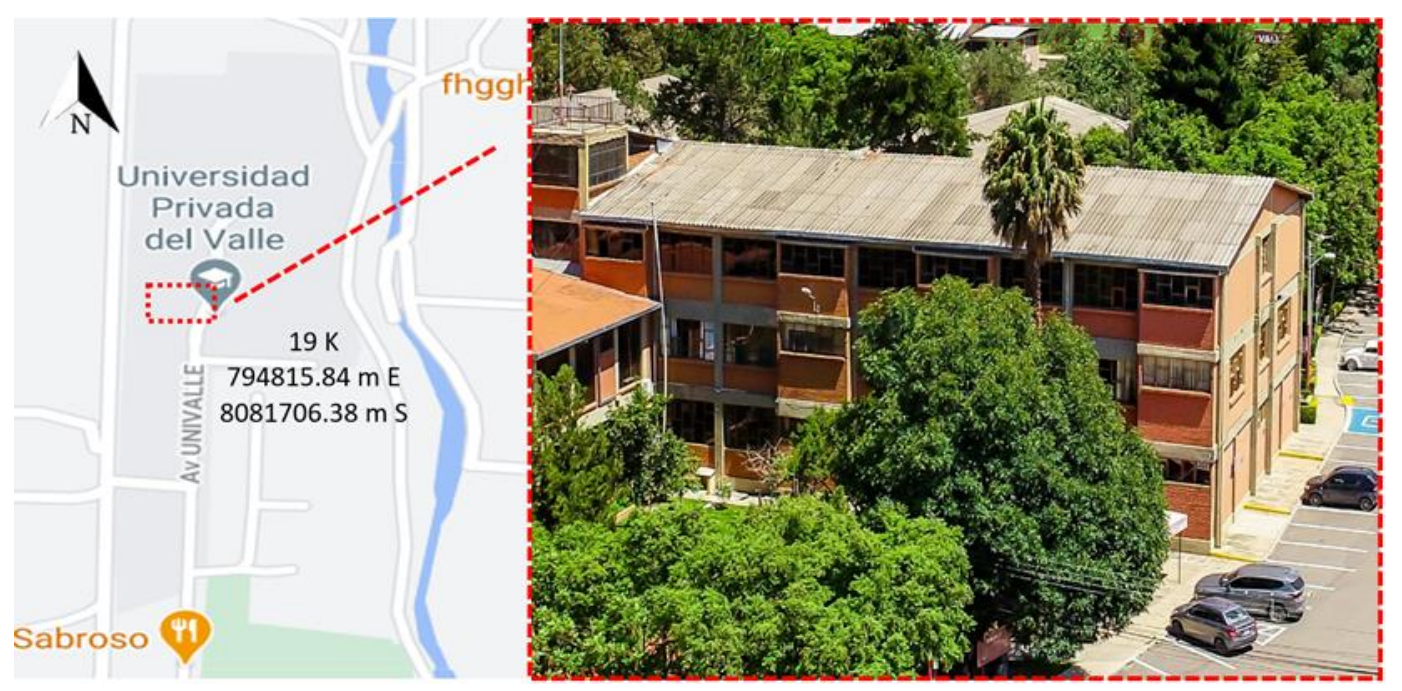

Figura 1 - Localización del Bloque E de la Universidad Privada del Valle

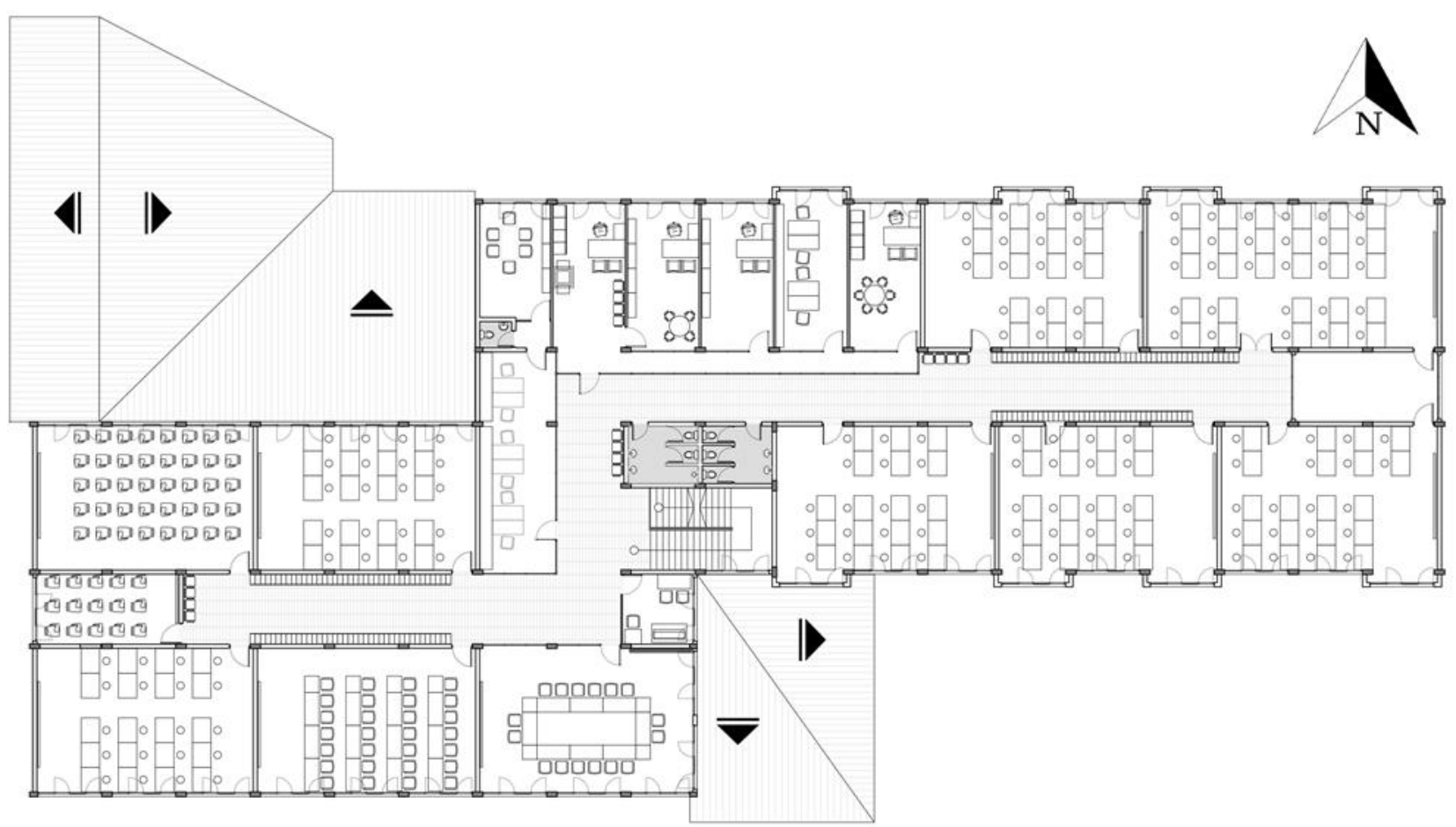

Figura 2 - Plano de la tercera planta del Bloque $\mathrm{E}$

La segunda etapa consistió en la inspección del área seleccionada por medio de un registro fotográfico y un checklist. Se elaboró el plano de la planta inspeccionada a fin de identificar las manifestaciones patológicas por ambiente, utilizando una leyenda específica. Esto se desarrolló con el mayor cuidado posible para evitar errores y repercusiones posteriores (Matellan, 2016).

Finalmente, en la tercera etapa se realizó un conteo de los tipos de manifestaciones patológicas presentes en los componentes de la edificación (cielo falso, revoque interior, pisos, etc), para luego asociar estos 
problemas con su origen dentro de las etapas del proceso constructivo. Estos resultados se presentaron en forma porcentual.

\section{Resultados y discusión}

Durante la inspección varias manifestaciones patológicas fueron identificadas, tales como: desprendimiento de cerámicas (Figura 3a), exposición y deterioro de tuberías (Figura 3b), fisuras en pisos de hormigón (Figura $3 c$ ), desprendimiento de zócalos (Figura 3d), desprendimiento del revoque de yeso (Figura 3e), deterioro y levantamiento de piso alfombrado (Figura 3f), daño de cielo falso (Figura 3g), deterioro de iluminación (Figura $3 \mathrm{~h})$, entre otros.
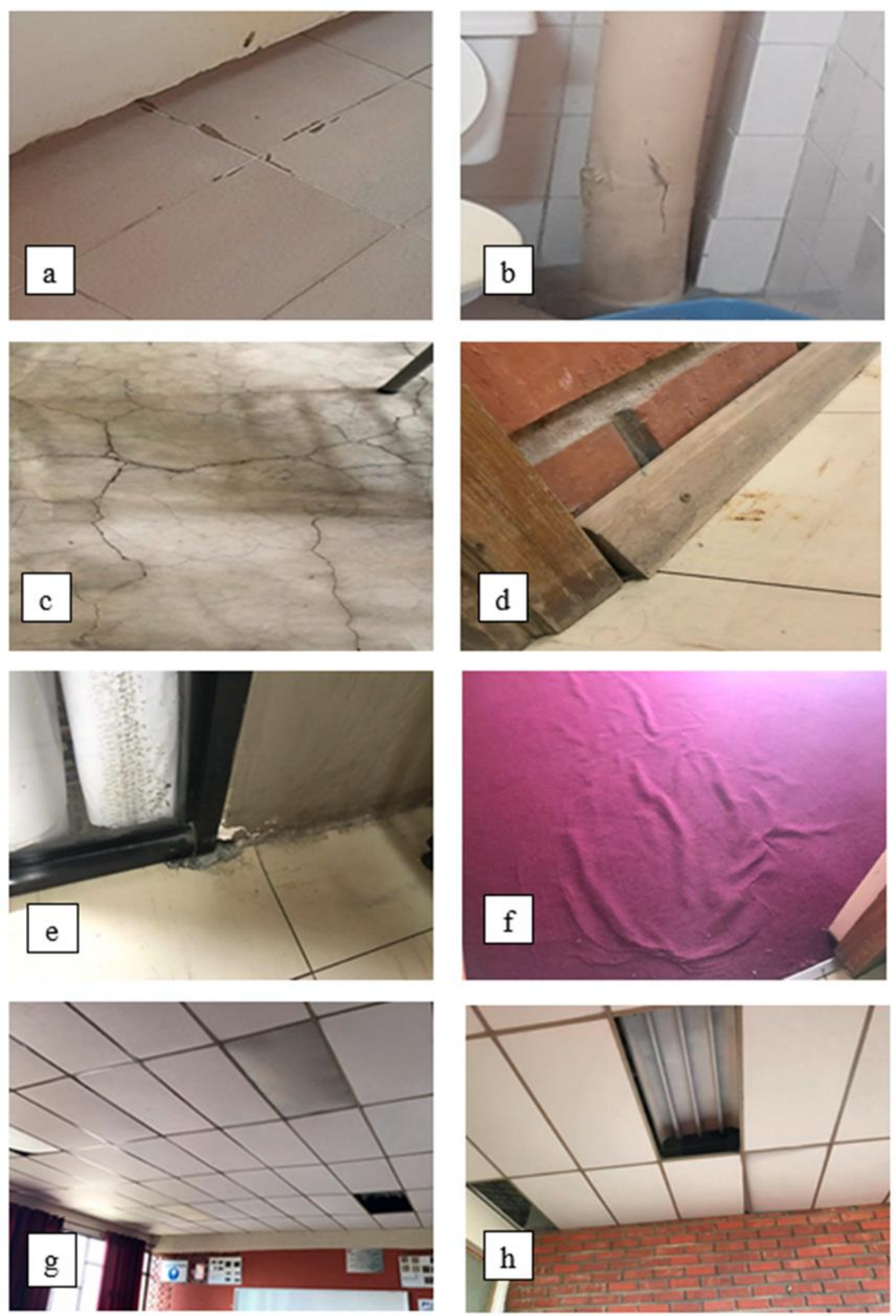

Figura 3 - Plano de la tercera planta del Bloque E

La Figura 4 muestra la ubicación de las manifestaciones patológicas por ambiente. Se puede observar que las áreas más afectadas son las aulas, oficinas, baños y pasillo. Resaltando, las oficinas y el pasillo con cinco problemas cada uno. 


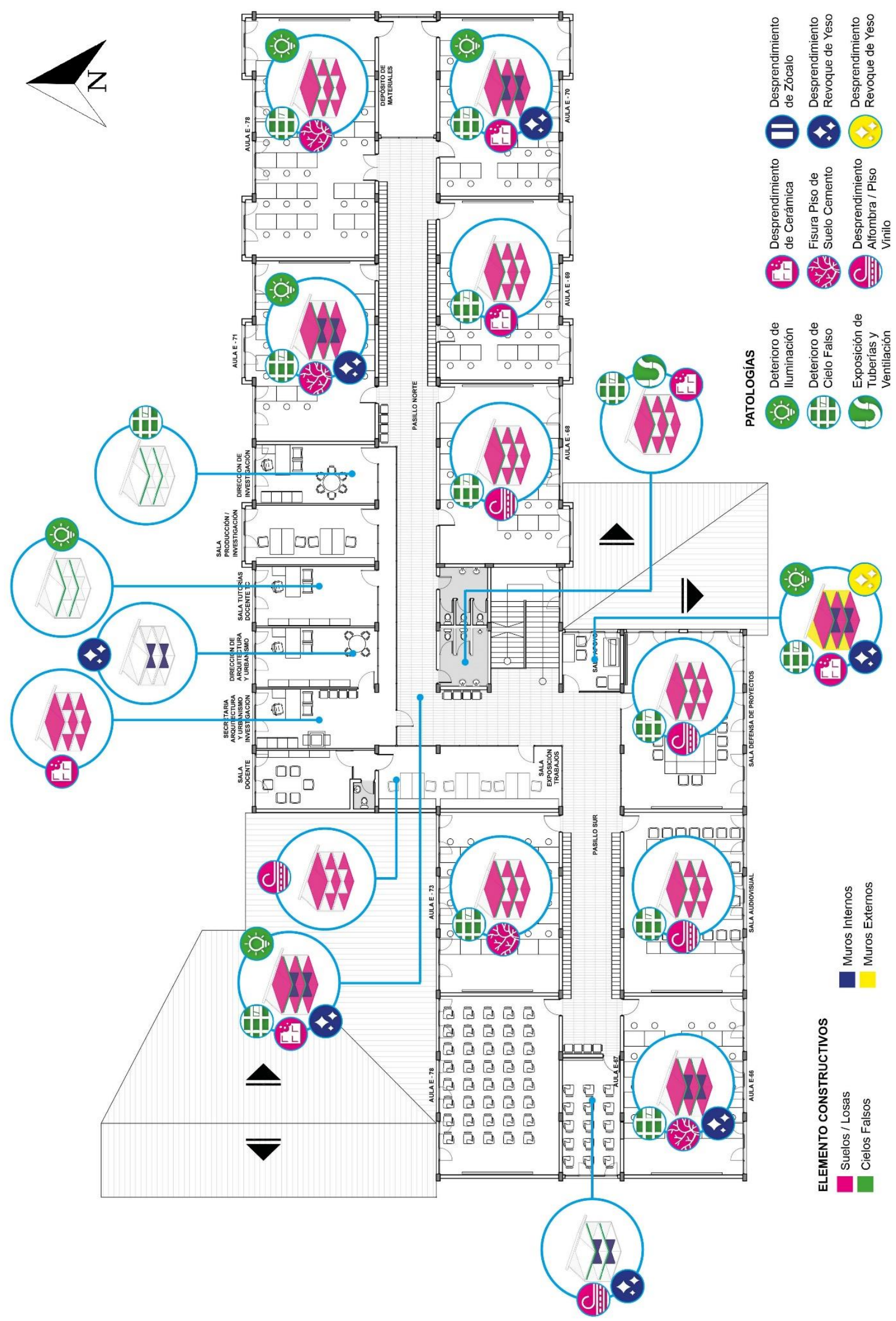

Figura 4 - Localización de las manifestaciones patológicas por ambiente 
Para determinar la manifestación patológica más frecuente, en la Figura 5 se presenta la incidencia de cada tipo de problema. El deterioro del cielo falso está presente en el $81.25 \%$ de los ambientes, seguido de desprendimiento de yeso con $43.75 \%$, deterioro de iluminación y desprendimiento de cerámica con $37.50 \%$, ambos. Las manifestaciones que menor frecuencia presentaron fueron la exposición de tuberías y desprendimiento de zócalos, ambas solo en un ambiente (6.25\%).

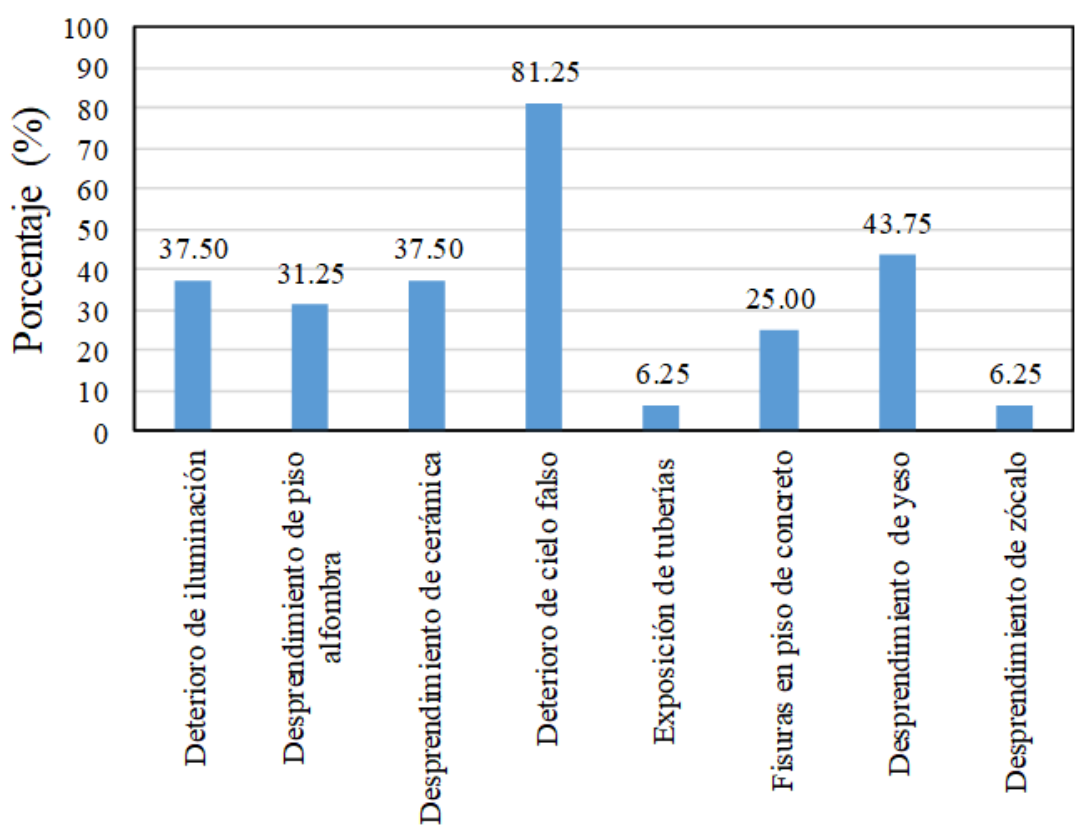

Figura 5 - Incidencia de las manifestaciones patológicas

El deterioro del cielo falso se debe principalmente a la falta de impermeabilización del techo, una vez que la planta inspeccionada se encuentra en el último nivel. El agua de lluvia se infiltra en la edificación, alcanzando el cielo falso, compuesto principalmente de placas de yeso, lo que genera deterioro. No obstante, también se comprobó que la infiltración llegó a afectar a las conexiones eléctricas, presentando daño en las luminarias. Es necesario verificar la impermeabilización del techo, corrigiendo los problemas, para luego cambiar las placas de yeso y luminarias afectadas.

El desprendimiento de yeso se debe tanto a la falta de adherencia con el muro de ladrillos como a las deficientes condiciones del colocado de marcos, ya que estos problemas se encuentran cerca de ventanas y puertas.

Durante la inspección se evidenció el desprendimiento de cerámicas que, en varios casos, fue posible retirarlas fácilmente. Esto indica que la cerámica no se adhirió al mortero, señalando que no se consideraron los tiempos de curado para este último. Los zócalos también presentaron el mismo problema. En este caso, se deben retirar las piezas de cerámica sueltas y realizar nuevamente el colocado con mortero nuevo, respetando los tiempos de curado para una adherencia adecuada.

Las fisuras son problemas encontrados comúnmente durante las inspecciones, estas pueden surgir por varias causas (Rocha et al., 2017). En la edificación se identificaron fisuras en los pisos de hormigón, lo que puede indicar que se producen por retracción plástica, relacionado con una cantidad elevada de cemento en la mezcla, problema normalmente encontrado en los pisos de hormigón (Romano et al., 2011).

La observación y el posterior análisis permiten determinar el origen y causas de las manifestaciones patológicas, que pocas veces se encuentran de manera evidente. En este sentido, la Figura 6 presenta el origen de las manifestaciones patológicas asociadas a una etapa del proceso constructivo. El uso y mantenimiento se presenta como el origen más recurrente, con $51.16 \%$, seguido por la ejecución (30.23\%), materiales $(16.28 \%)$, proyecto $(2.33 \%)$ y planeamiento $(0 \%)$. 


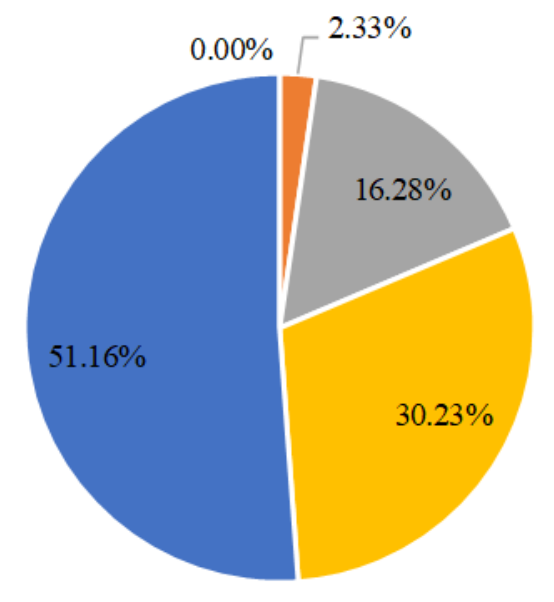

- Planeamiento | Proyecto " Materiales $\mid$ Ejecución $\mid$ Uso y mantenimiento

Figura 6 - Origen de las manifestaciones patológicas relacionadas al proceso constructivo

Los defectos pueden tener origen en cualquier etapa del proceso constructivo y se relacionan con el control de calidad de las diversas etapas constructivas (Cremonini, 1988). En este caso, por la edad del Bloque E y la falta de mantenimiento periódico, la etapa de uso tiene la mayor incidencia en los problemas identificados. Helene (1992) señala que, de las cinco etapas consideradas, el uso y mantenimiento tiene el mayor periodo de tiempo, puesto que las estructuras se diseñan, en general, para más de 50 años. Esto último puede llevar al surgimiento de problemas con el tiempo. Con relación a la etapa de ejecución, es necesario un control de calidad adecuado, respetando todos los procedimientos establecidos y recomendados, una vez que se presentó una elevada incidencia de defectos en esta etapa.

Si bien las manifestaciones patológicas encontradas no representan un riesgo estructural para la edificación ni sus elementos, significan un perjuicio funcional y estético, comprometiendo el desempeño de la estructura y generando mayor deterioro con el tiempo. Es recomendable una intervención a corto plazo para garantizar la vida útil proyectada, evitando estados avanzados de daño y elevados costos.

\section{Conclusiones}

A través de las herramientas utilizadas se lograron identificar las manifestaciones patológicas en la tercera planta del Bloque $\mathrm{E} y$, con un mapeo de problemas, se detectaron las áreas más comprometidas (oficinas y pasillo).

Se recomienda tener registros fotográficos de la edificación a lo largo de su vida útil, con el objetivo de evaluar la evolución de los distintos problemas y dar diagnósticos más certeros en el futuro.

En el presente estudio se demostró que, el uso y mantenimiento, es el origen más frecuente de los problemas. Esta etapa debe ser considerada importante para que los entes encargados provean programas de mantenimiento y uso acordes a los proyectados.

Las actividades de inspección y mantenimiento son necesarias a fin de recuperar las áreas afectadas, puesto que, los problemas detectados pueden agravarse con el tiempo y pasar de ser estéticos a estructurales.

\section{Referencias Bibliográficas}

American Society for Testing and Materials (1981). ASTM E 632-81: Standard Practice for Developing Accelerated Tests to a Prediction of the service Life of Building Components and Materials. Philadelphia: United States, American Society for Testing and Materials.

Antunes, G.R. (2010). Estudo de manifestações patológicas de fachadas em Brasília - Sistematização da incidência de casos. Disertación de Maestría. Facultad de Ingenieria, Universidad de Brasilia, Brasil. 
Cánovas, M.F. (1994). Patología y terapéutica del hormigón armado. Madrid, España: Colegio de Ingenieros de Caminos, Canales y Puertos, Servicio de Publicaciones.

Cremonini, R.A. (1988). Incidência de manifestações patológicas em unidades escolares da região de Porto Alegre: Recomendações para projeto, execução e manutenção. Disertación de Maestría. Universidade Federal do Rio Grande do Sul.

Harris, S.Y. (2001). Building Pathology. Deterioriration, disgnostics and Intervention. New York, United States: Jhon Wiley \& Sons.

Helene, P. (1992). Manual de reparo, proteção e reforço de estruturas de concreto. São Paulo, Brasil: PINI.

Helene, P. R. (2015). Manual para reparación, refuerzo y protección de las estructuras de concreto. México D.F., México: IMCYC.

Husni, R. (2007). Fallas y prevención en las estructuras de hormigón. Revista Ingeniería Estructural, 15 (39).

Matellan, M. (2016). Colección de planos, planoteca. Quito, Ecuador: Cediza Editorial

Ministerio de Obras Públicas, Servicios y Vivienda (2006). Norma Boliviana de Diseño Sísmico. La Paz, Bolivia: MOPSV.

Oliveira, D.F. (2013). Levantamento de causas de patologias na construção civil. Trabajo de Conclusión de Curso. Escola Politécnica Universidade Federal do Rio de Janeiro, Brasil.

Pallarés, H.P. (2007). Sobre tipos de edificios. Quaderns d'arquitectura i urbanisme, 256, 136-141.

Quintero-Quintero, M.I., Solano-López, C.A., Pandales-Lozano, C.A. (2013). La degradación y el mantenimiento en las obras de edificación: estudio de caso Institución Educativa Antonio Derka Santo Domingo. Facultad de Ingenierías, Universidad de Medellín, Colombia.

Rocha, J.H.A., Silva, M., Póvoas, Y., Monteiro, E. (2017). Análise da Profundidade de Fissuras em Concreto com Termografia Infravermelha. Revista de Engenharia e Pesquisa Aplicada, 2(3). 58-65.

Romano, R.C., Cardoso, F.A., Pilleggi, R.G. (2011). Propriedades do Concreto no Estado Fresco. In Concreto: Ciência e tecnologia, São Paulo, Brasil: IBRACON.

Silva, C. D., Coelho, F., de Brito, J., Silvestre, J., and Pereira, C. (2017). Inspection, diagnosis and repair system for architectural concrete surfaces. J. Perform. Constr. Facil., 31(5).

Tutikian, B., Pacheco, M. (2013). Inspección, diagnóstico y pronóstico en la construcción civil. Mérida, México: ALCONPAT. 